\title{
Body Tracking MDV: sistema aplicado para fisioterapia com uso de tecnologias de controle por gestos
}

\author{
Marcos D. Vendramini ${ }^{1}$, Paulo Augusto Nardi ${ }^{1}$, Alexandre R. Paschoal ${ }^{1 *}$ \\ ${ }^{1}$ Mestrado Profissional em Informática (PPGI), \\ Lab. de Jogos Digitais (UTFGames), Depto. de Computação,
} Universidade Tecnológica Federal do Paraná (UTFPR), Cornélio Procópio - PR - Brasil

*paschoaleutfpr.edu.br

\begin{abstract}
This article presents a program for the area of physiotherapy implemented with gesture control technologies, mapping the patient's body and tracking the exercise during its execution. The basis of its functioning is in the system of validation of the exercise by comparing angles and references of the body points, with models of correct movements previously stored. The program also provides feedback on patient evolution, comparing movements of the same exercise throughout the treatment. The project aims at helping the physiotherapist to monitor the patients' performance. Body Tracking MDV is available, including its source code at: http://www.marcosdiasvendramini.com.br/mestrado/.
\end{abstract}

Resumo. Este artigo apresenta um programa para área da fisioterapia implementado com tecnologias de controle por gestos, mapeando o corpo do paciente e rastreando o exercício durante sua execução. A base do seu funcionamento está no sistema de validação do exercício por comparação de ângulos e referências dos pontos corporais, com modelos de movimentos corretos previamente armazenados. O programa fornece, também, o feedback da evolução do paciente, comparando movimentos de um mesmo exercício ao longo do tratamento. O projeto visa a auxiliar o fisioterapeuta no monitoramento dos exercícios executados pelos pacientes. O Body Tracking MDV está disponível, inclusive seu código fonte em: http://www.marcosdiasvendramini.com.br/mestrado/.

\section{Introdução}

Jogos sérios são aplicados na Saúde, em atividades terapêuticas e físico-motoras, com o objetivo de motivarem o paciente na execução de exercícios de reabilitação e de fornecerem constante feedback tanto para paciente como para terapeuta [Junior et al. 2013]. De acordo com [Clua 2014], o uso de dispositivos de captura de movimento potencializam a aplicação de tais jogos.

Dentre as tecnologias de captura de movimento está a de controle por gestos [Caprani et al. 2012], na qual o movimento corporal é capturado em tempo real [Chan et al. 2011] com o uso de sensores de movimento. Atualmente, estes sensores estão presentes em sistemas de entretenimento doméstico cuja popularização os tornou economicamente acessíveis, a exemplo do Kinect ${ }^{1}$, ainda, tais equipamentos não são

\footnotetext{
${ }^{1}$ Kinect é um sensor de movimento, que sincroniza cor e profundidade de imagens, possibilitando interações entre jogadores e aplicação sem a necessidade de controles [Han et al. 2013]. Desenvolvido pela PrimeSense e posteriormente adquirido pela Microsoft foi incorporado como periférico do console Xbox 360 [Webb and Ashley 2012].
} 
intrusivos, o que torna fácil a aplicação em conjunto com aplicativos de reabilitação [Liu et al. 2013], como na fisioterapia.

Conforme revisão feita neste artigo (veja Tabela 1), apesar de existirem diversos aplicativos para fisioterapia, poucos são de código aberto, o que impede ou dificulta o reúso em novos tratamentos que necessitem adaptações ou extensões de suas funcionalidades.

Neste artigo, é apresentado um software de código aberto com tecnologia de controle por gestos para aplicações em atividades físicas e de reabilitação. O software Body Tracking MDV está disponível em: http://www.marcosdiasvendramini.com.br/mestrado/, sob a licença GNU GPL (General Public License) ${ }^{2}$. A aplicação permite a captura dos movimentos do paciente, compara os dados extraídos com uma base de movimentos modelos previamente cadastrados e apresenta os resultados por meio de gráficos. A contribuição deste projeto foi a de disponibilização de um programa base de captura e comparação de movimento que possa ser reusável em conjunto com outras aplicações na área da Saúde, como ferramenta para ajudar em diagnóstico, ou em Jogos Sérios de Reabilitação.

O restante do artigo está estruturado da seguinte forma: na Seção 2, são apresentados os trabalhos relacionados; na Seção 3, é descrito o desenvolvimento e a arquitetura do sistema proposto; na Seção 4, é ilustrado um exemplo do sistema em execução e suas principais funcionalidades; e, na Seção 5, são apresentadas as considerações finais.

\section{Trabalhos Relacionados}

Foi realizada uma revisão bibliográfica dos artigos no período de 2010 até 2017 usando os termos Rehabilitation Kinect, Gesture Rehabilitation, Rehabilitation Kinect Angle, Reabilitação Kinect, Reabilitação Sensor Movimento e Controle Gestos Reabilitação como string de pesquisa. A busca foi executada nas bases de dados da IEEE Xplore Digital Library e Google Scholar. Removeram-se os artigos que não apresentavam: área da saúde e tecnologias de gesto.

Como resultado, dez artigos foram selecionados (Tabela 1). Destes, nove dos trabalhos usam Kinect, e um utiliza o Wii Remote ${ }^{3}$ [Romano et al. 2013].

O PhysioJoy [Balista 2013] utiliza o Kinect em três serious games comerciais que tratam de um tipo específico de lesão corporal ou cerebral, validando o movimento de membros superiores e inferiores por meio da localização de pontos. O Fisiogame [Fernandes et al. 2014] é um jogo com realidade aumentada/virtual para execução de exercícios de fisioterapia. Este reconhece o posicionamento do jogador mostrando na tela se o movimento está ou não sendo executado de forma correta. O jogo valida o movimento de membros superiores por meio de comparação por ângulos. O KSGphysio [Duarte et al. 2014] está desenvolvendo um jogo que faz a validação do movimento de membros superiores e inferiores por meio da comparação de ângulos, permitindo que o

\footnotetext{
${ }^{2}$ GNU General Public License ou Licença Pública Geral, é a designação da licença para software livre idealizada por Richard Matthew Stallman em 1989, no âmbito do projeto GNU da Free Software Foundation [Campos 2006].

${ }^{3} \mathrm{O}$ Wii remote é o controle do console Wii, ele capta os movimentos realizados pelo usuário ao movê-lo, por meio de três acelerômetros embutidos e de uma câmera infravermelho [Sousa 2011].
} 
Tabela 1. Comparativo de trabalhos relacionados

\begin{tabular}{lcllcccc}
\hline \multicolumn{1}{c}{ Nome } & Ano & Dispositivo & Validação & MS & MI & PE & Licença \\
\hline $\begin{array}{l}\text { SeeMe } \\
\text { Rehabilitation }\end{array}$ & 2011 & Kinect & $\begin{array}{l}\text { Não } \\
\text { Informado }\end{array}$ & X & X & X & Comercial \\
\hline MIRA Rehab & 2011 & Kinect & $\begin{array}{l}\text { Não } \\
\text { Informado }\end{array}$ & X & & & Comercial \\
\hline Saini et al. 2012 & 2012 & Kinect & Ângulos & X & X & Código aberto \\
\hline PhysioJoy & 2013 & Kinect & $\begin{array}{l}\text { Localização } \\
\text { dos pontos }\end{array}$ & X & X & & Comercial \\
\hline $\begin{array}{l}\text { Romano et al. 2013 } \\
\text { LabView }\end{array}$ & 2013 & $\begin{array}{l}\text { Wii } \\
\text { Remote }\end{array}$ & $\begin{array}{l}\text { Localização } \\
\text { dos Pontos }\end{array}$ & & & X & Comercial \\
\hline NeuroAtHome & 2013 & Kinect & $\begin{array}{l}\text { Não } \\
\text { Informado }\end{array}$ & X & X & X & Comercial \\
\hline KSGphysio & 2014 & Kinect & Ângulos & X & X & & $\begin{array}{l}\text { Fase de } \\
\text { pesquisa }\end{array}$ \\
\hline Fisiogame & 2014 & Kinect & Ângulos & X & & $\begin{array}{l}\text { Fase de } \\
\text { pesquisa }\end{array}$ \\
\hline Sousa et al. 2016 & 2016 & Kinect & Ângulos & X & X & X & $\begin{array}{l}\text { Fase de } \\
\text { pesquisa }\end{array}$ \\
\hline Estepa et al. 2016 & 2016 & Kinect & Ângulos & & & X & $\begin{array}{l}\text { Fase de } \\
\text { pesquisa }\end{array}$ \\
\hline
\end{tabular}

MS: Membros Superiores. MI: Membros Inferiores. PE: Postura e Equilíbrio.

usuário defina a velocidade e o nível de dificuldade, adequando-se a condição do paciente para a execução do movimento.

Em [Romano et al. 2013], foi desenvolvido um software utilizando o LabVIEW, com o objetivo de demonstrar a adaptação deste software para leitura e quantificação dos dados gerados a partir do acessório Wii Balance Board. O foco das atividades é a avaliação do equilíbrio estático do paciente, utilizando a localização por pontos para validação da postura e equilíbrio corporal. O LabVIEW é um ambiente de desenvolvimento integrado, criado especificamente para engenheiros e cientistas, que oferece um conjunto abrangente de ferramentas que permite desenvolver qualquer aplicação de medição ou controle de forma rápida. O LabVIEW permite uma fácil programação em blocos com interação entre diversos tipos de softwares e plataformas, sendo possível organizar os dados de entrada e saída do projeto da melhor forma para a visualização e manipulação destes [Instruments 2017]. Em [Sousa et al. 2016], foi apresentado um software para fisioterapia que grava uma pose padrão para o exercício e compara com a pose executada pelo paciente. Para efetuar a comparação, o autor utiliza a angulação das articulações. Em [Estepa et al. 2016], também é realizada a comparação dos ângulos das articulações para validar os exercícios. $\mathrm{O}$ autor utiliza jogos sérios para o tratamento de postura e equilíbrio dos pacientes.

SeeMe Rehabilitation

[Rehabilitation 2017], NeuroAtHome [Neuroathome 2016] e MIRA Rehab [Mira 2016] são jogos comerciais que utilizam o Kinect para a validação dos movimentos, mas a forma de validação não foi informada por nenhum dos autores. Em [Rehabilitation 2017] e [Neuroathome 2016] 
são validados os exercícios dos membros superiores, membros inferiores e de postura e equilíbrio corporal, enquanto em [Mira 2016], são validados apenas os exercícios de membros superiores. Em [Saini et al. 2012], foi desenvolvido um jogo de código aberto que valida movimentos de membros superiores e membros inferiores, por meio da comparação dos ângulos das articulações.

Diferente dos trabalhos relacionados, o software apresentado neste artigo não se restringe a um tipo de exercício, sendo possível validar e analisar os membros superiores, membros inferiores, postura e equilíbrio. Ainda, o software oferece um cadastro completo de novos exercícios, permitindo ao fisioterapeuta adicionar novas validações. A restrição do software relaciona-se à oclusão de membros, pois o Kinect não capta a localização dos pontos que estejam obstruídos. Deste modo, se um ponto, como o da mão, passar por trás do corpo, sua localização se perderá pelo período em que estiver ocluso.

Dos trabalhos mencionados, apenas o trabalho dos autores [Saini et al. 2012] possui licença de código aberto, os trabalhos dos autores [Sousa et al. 2016], [Estepa et al. 2016] e os softwares KSGphysio, Fisiogame ainda estão em fase de desenvolvimento, não estando disponível para o público. Os demais trabalhos estão disponíveis, porém com licença comercial.

\section{Body Tracking MDV: Desenvolvimento e arquitetura}

Para o captura de movimentos, foi usado o Kinect 360, por apresentar melhor custobenefício entre os dispositivos disponíveis no mercado e por atender às necessidades do projeto, como: reconhecimento de pontos por todo o corpo e alcance de até $400 \mathrm{~cm}$ de profundidade - o que permite uma área de atuação satisfatória.

$\mathrm{Na}$ Figura 1, é esquematizada a aplicação da tecnologia de controle por gestos. $\mathrm{O}$ dispositivo captura a imagem e os pontos do corpo da pessoa; os dados são enviados para o sistema e armazenados para posterior validação dos movimentos, ou para ser cadastrado como exercício padrão e servir como modelo para a validação do movimento.

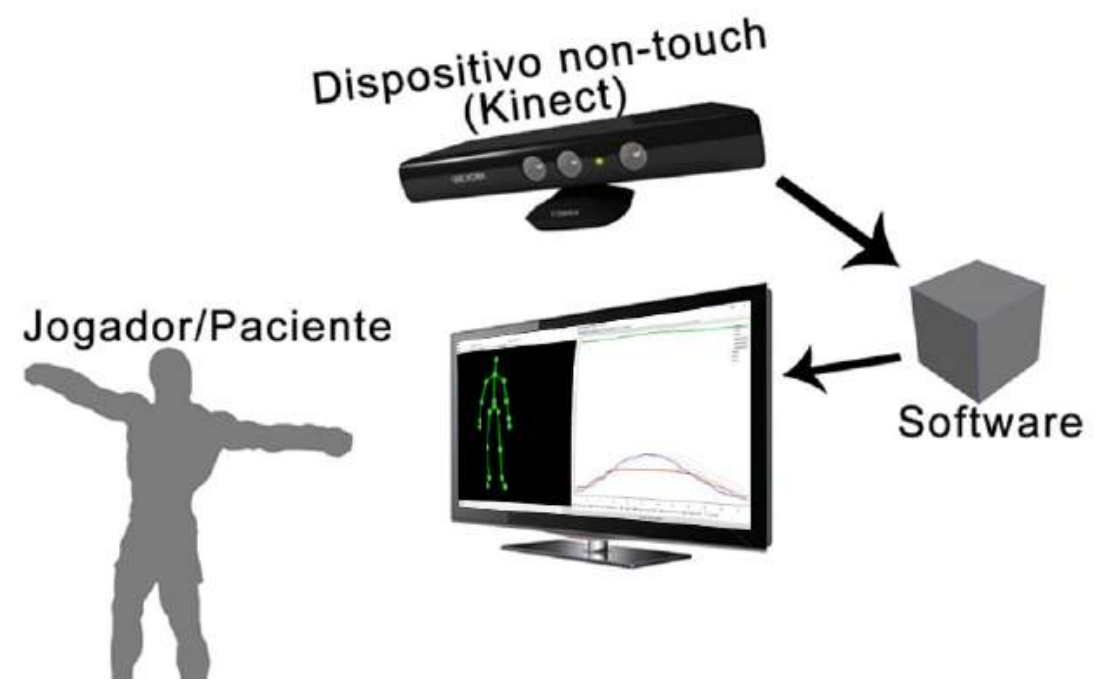

Figura 1. Aplicação da tecnologia de controle por gestos (Adaptado e atualizado de [Vendramini et al. 2015]) 
Para o desenvolvimento do software, foi utilizada a IDE (Integrated Development Environment / Ambiente de Desenvolvimento Integrado) Visual Studio Community 2015 da Microsoft. A programação da interface gráfica foi desenvolvida na Windows Presentation Foundation (WPF), e as funcionalidades do software foram programadas na linguagem de programação C\#. O pacote Visual Studio foi obtido gratuitamente em sua versão Community no site da Microsoft. Para a captura de movimentos, foi utilizada a biblioteca "Microsoft.Kinect", parte da versão 1.8 da SDK do Kinect 360.

Os dados da captura dos movimentos são convertidos pelo sistema em registros no formato JSON (JavaScript Object Notation / Notação de Objetos JavaScript). Cada registro representa os dados de cada um dos pontos corporais, contendo a posição de cada ponto na horizontal $(\mathrm{X})$, vertical $(\mathrm{Y})$ e profundidade $(\mathrm{Z})$.

\section{Funcionalidades e aplicações}

Atualmente o sistema possui seis exercícios cadastrados: um de elevação lateral do braço esquerdo; um de elevação lateral do braço direito; um de elevação lateral com os dois braços simultâneos; um para o joelho esquerdo; um para o joelho direito; e, um para a postura da coluna vertebral. Cada exercício cadastrado possui uma base de dados de modelos com várias execuções do exercício.

O sistema permite o cadastramento de novos movimentos modelos, isto é, aqueles corretamente executados e que servirão como base de comparação para os exercícios realizados pelos pacientes. A seleção dos movimentos modelos e seu cadastramento são realizados manualmente por um profissional responsável pela operação do software, na tela de cadastro de exercícios/movimentos Figura 2. Nesta tela, o profissional deve informar qual o nome do exercício e o caminho físico dos dados do exercício modelo, selecionando a pasta salva com o nome do movimento informado na tela de captura.

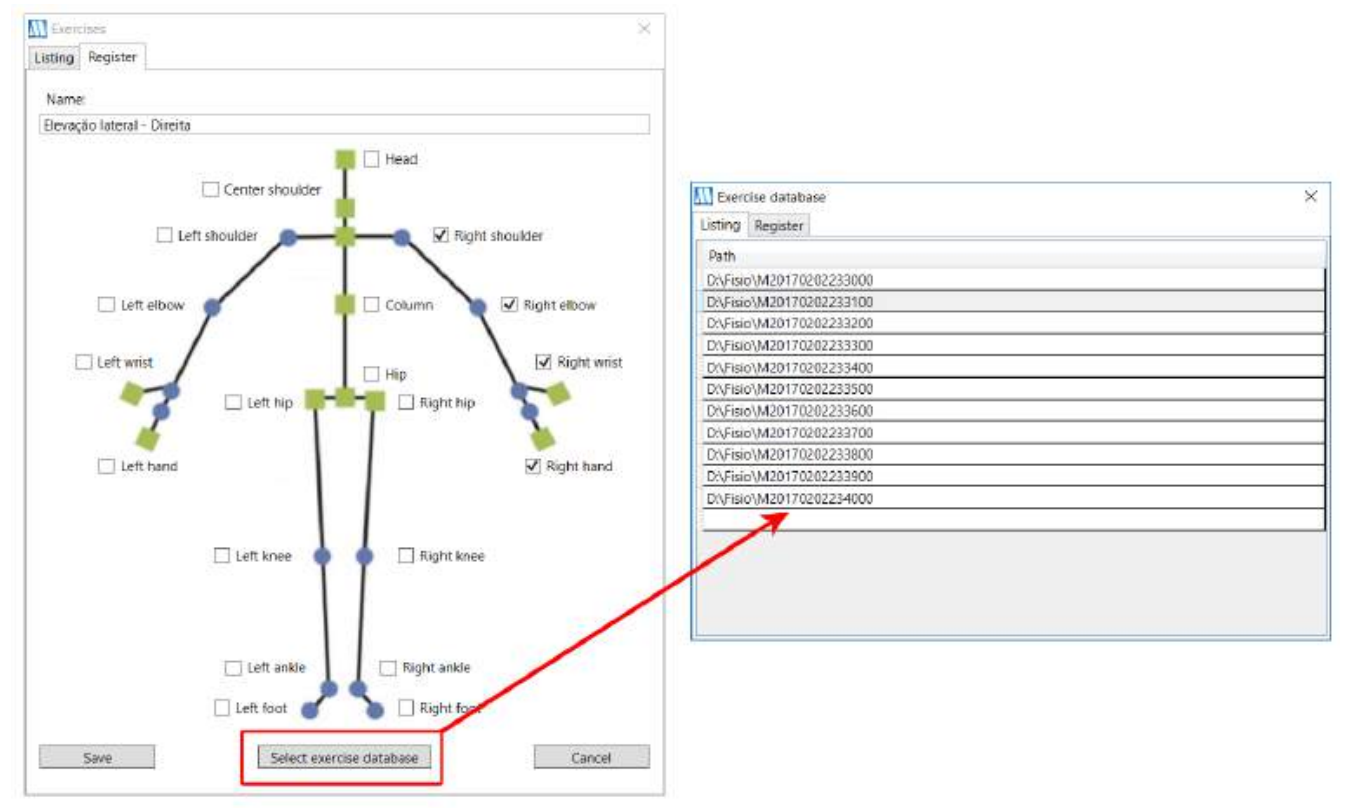

Figura 2. Captura dos dados com personalização dos pontos e itens cadastrados

Ao iniciar a utilização do sistema para a realização do exercício, o profissional 
deve cadastrar o paciente com os seguintes dados: nome, data de nascimento e, opcionalmente, observação.

A configuração da captura dos dados do paciente é realizada por meio de uma tela, como na Figura 3. Nesta etapa, deve-se escolher o nome do paciente e o exercício ou movimento que será executado. Também é possível efetuar configurações visuais, como mostrar a pessoa na tela, mostrar as ligações e articulações, e se o vídeo do exercício deve ser salvo. Todos os dados obtidos pela captura são salvos em um arquivo JSON, conforme mencionado anteriormente.

Caso a opção de salvar o vídeo da pessoa esteja habilitada, o sistema armazena também a imagem da pessoa em formato PNG (Portable Network Graphics), sincronizada com a posição dos pontos do corpo salvos no JSON. Deste modo, para cada registro, é salva uma imagem do paciente. De acordo com [Microsoft 2017], o Kinect 360 possui uma taxa de atualização de trinta quadros por segundo, gerando assim trinta registros no arquivo JSON com as referências dos pontos corporais, e caso a opção de salvar o vídeo da pessoa esteja habilitada, trinta imagens do paciente são armazenadas, por segundo.

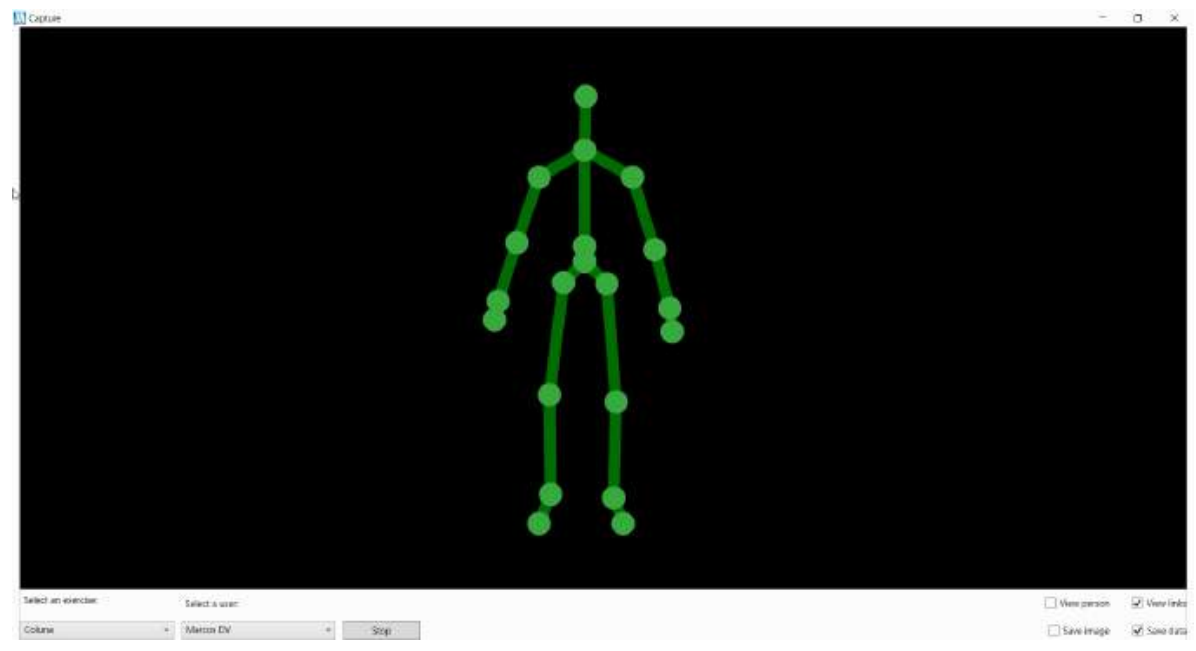

Figura 3. Tela de captura dos dados do exercício

Após a captura de um movimento, o software apresenta os dados extraídos na forma de gráfico (Figura 4) para análise do profissional. Caso a opção de gravar o movimento em vídeo seja habilitada, este é apresentado à esquerda do gráfico. O software permite a opção de avançar ou retroceder a execução do movimento gravado.

Para iniciar a análise, o profissional deve escolher o paciente e o exercício a ser analisado. O software mostra todo o movimento que foi coletado na tela de captura e apresenta uma tela com todos os dados do movimento em forma de gráfico. Nesta tela, é possível escolher qual ponto do corpo será analisado. Uma vez definido o ponto do corpo, o software monta um gráfico gerado com os dados do movimento. Os gráficos acompanham o movimento do paciente que está sendo exibido na tela de análise, facilitando a visualização dos dados do movimento.

Este gráfico, exibido em destaque na Figura 5, compara o movimento realizado pelo paciente com base nos movimentos modelos para um exercício específico, gerando uma média e um desvio padrão do movimento. O gráfico mostra a variação do movimento 


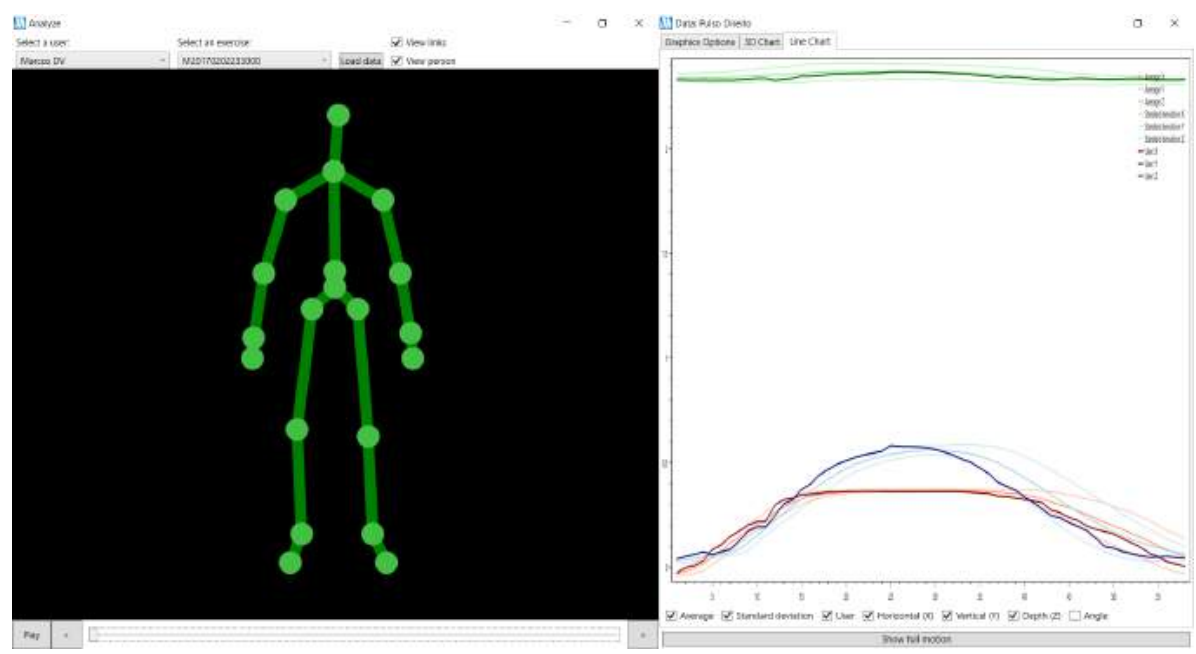

Figura 4. Análise do movimento do paciente com a movimentação e o gráfico do exercício

do usuário, com a variação do ponto na horizontal (X - linha de cor vermelho escuro continuo), vertical ( $Y$ - linha azul continua), e profundidade ( $\mathrm{Z}$ - linha verde escuro continua). Em destaque na Figura 5, há a linha azul (Y), a média (linha em azul claro continuo) e desvio padrão do movimento (linha azul claro pontilhada). As cores da média e desvio padrão seguem um padrão, sempre mostrando a mesma cor da linha do movimento do usuário, porém em um tom mais claro, e a linha do desvio padrão sempre pontilhada.

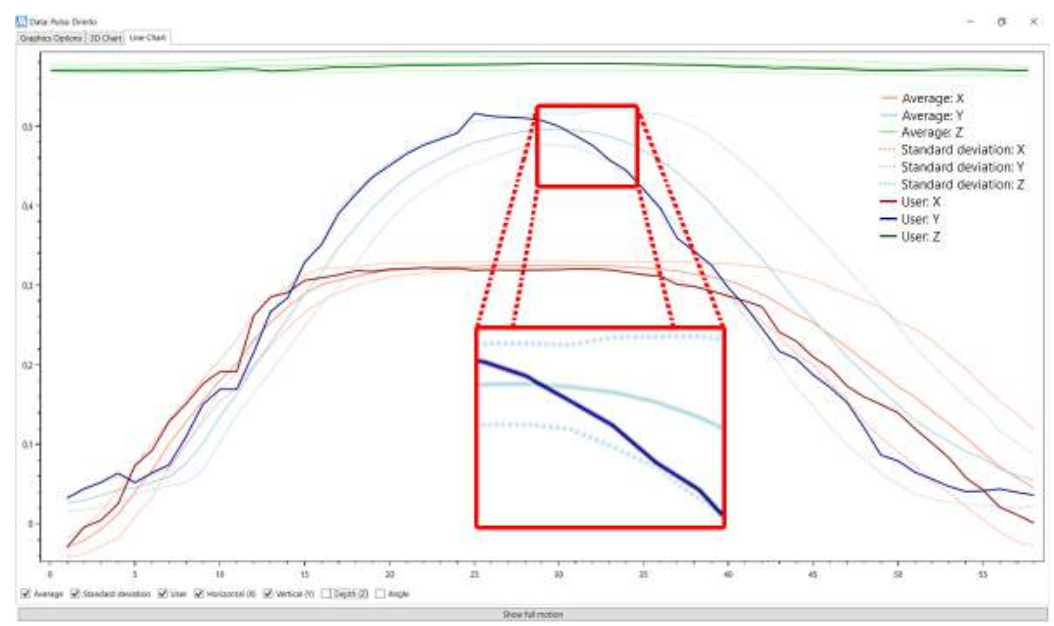

Figura 5. Gráfico do exercício com a variação $X, Y$ e $Z$ do pulso direito. Em destaque: A linha do paciente (Azul escuro) com a média (Azul claro) e desvio padrão (Azul claro pontilhado) do exercício

Neste gráfico, também é possível exibir a variação da angulação da articulação escolhida. O software sempre calcula e apresenta a média e desvio padrão, tanto para os três eixos de movimento do paciente (X, Y e Z), como para o gráfico da variação dos ângulos de uma articulação. Para calcular o ângulo da articulação, utiliza-se a articulação escolhida para a análise e a articulação anterior e posterior. Por exemplo, para calcular o ângulo do cotovelo, são utilizados os pontos do pulso, do cotovelo e do ombro do paciente.

O softwate também possui uma tela que gera gráficos em 3D com o movimento 
de forma tridimensional (Figura 6). Esta tela possui dois gráficos: a Figura 6-A apresenta o movimento e sua variação realizado pelo paciente, enquanto que a Figura 6-B mostra a média gerada pela base de dados de exercícios corretos.

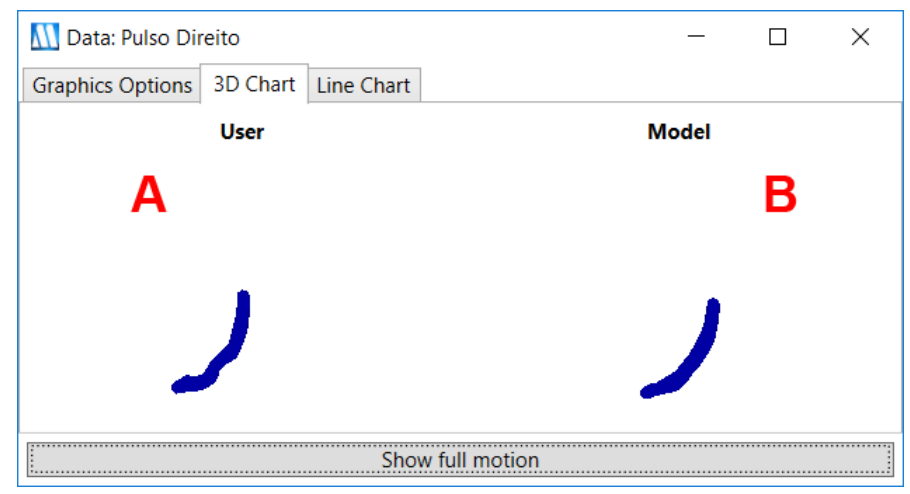

Figura 6. Tela com gráfico 3D do exercício: A- indica movimento do paciente e B- indica a média dos exercícios

O software também possui uma tela de comparação da evolução do paciente, a seleção é feita por meio de uma listagem dos movimentos já executados e salvos do paciente, a Figura 7 ilustra a seleção dos dados para iniciar a comparação. Esta tela oferece os mesmos recursos que a tela de análise tradicional (Figura 4), mas nesse caso, em vez de se comparar com a base de dados de movimentos corretos de um determinado exercício, comparam-se apenas dois exercícios do mesmo paciente, permitindo observar e comparar a variação de uma para o outro. Os gráficos gerados mostram, neste caso, a variação dos dois exercícios, tanto para a variação do ponto nos três eixos de movimento do paciente (X, Y e Z), como na variação da angulação da articulação. Para a comparação da evolução do paciente, também é possível gerar o Gráfico 3D, mostrando o movimento anterior e atual de forma tridimensional.

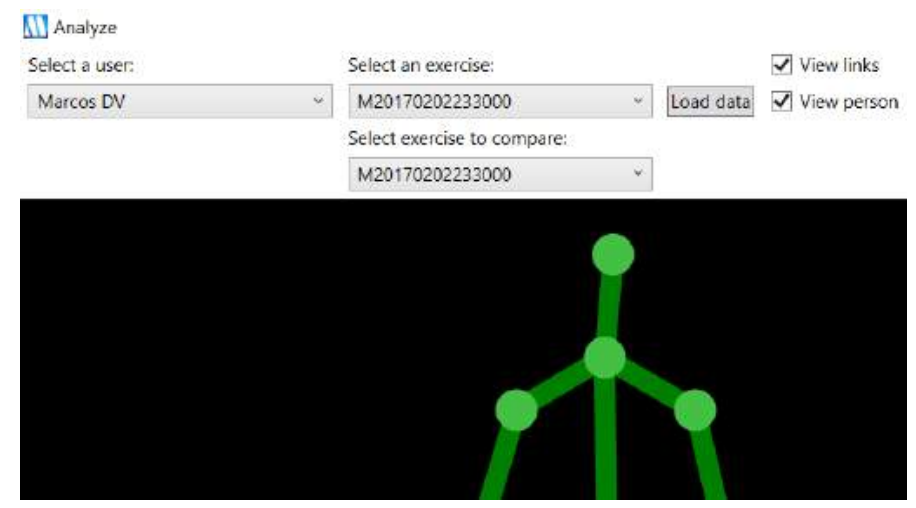

Figura 7. Tela de análise para comparar dois movimentos

\section{Considerações Finais}

O software desenvolvido permite que o profissional da saúde monitore e acompanhe a execução de exercícios físicos de reabilitação fisioterápica do paciente. Os exercícios são comparados com movimentos modelos disponíveis; a média e desvio padrão, para cada exercício, são gerados e apresentados na forma de gráfico para posterior análise 
do profissional. Também é possível acompanhar a evolução do paciente, comparando-se dois exercícios do mesmo tipo, realizados em períodos distintos. São considerados os movimentos dos membros superiores, membros inferiores, postura e equilíbrio.

Durante o processo de comparação, são analisados os ângulos e referências das articulações. Esta característica faz com que o sistema não se limite a um conjunto específico de tipo de exercício, de modo que diferentes atividades físicas possam ser analisadas. Este é um diferencial deste trabalho em relação aos descritos na Seção 2, que se restringem a um tipo único de exercício.

Como contribuição, o software apresentado auxilia o fisioterapeuta nos tratamentos de reabilitação, fornecendo parâmetros dos exercícios realizados pelos pacientes em comparação com execuções consideradas corretas, assim como comparações entre exercícios do mesmo paciente ao longo do tratamento.

O software apresentado tem a característica de ser expandido e alterado para que seu núcleo - a captura de movimentos e comparação com movimentos modelos - seja reusado em aplicações de diversas finalidades, como em: jogos sérios para reabilitação e em ferramentas de diagnóstico automatizado.

Por ser desenvolvido em código aberto, espera-se que a disponibilização do software contribua com a redução do trabalho de grupos de pesquisa que precisem de um software de captura de movimentos e comparação para finalidades diversas, como as anteriormente citadas.

A restrição do software relaciona-se com a oclusão de membros, pois o Kinect não capta a localização dos pontos que estejam obstruídos. Como trabalhos futuros, espera-se adicionar novos exercícios previamente cadastrados, melhorar o sistema de captura, com o objetivo de diminuir os pontos de oclusão e melhorar a precisão na captura dos dados pelo Kinect.

\section{Referências}

Balista, V. G. (2013). Sistema de realidade virtual para avaliação e reabilitação de déficit motor. SBGames. São Paulo, pages 16-20.

Campos, A. (2006). O que é software livre. BR-Linux. Florianópolis, março de.

Caprani, N., O'Connor, N. E., and Gurrin, C. (2012). Touch screens for the older user. In Assistive Technologies, pages 95-118. InTech.

Chan, J. C., Leung, H., Tang, J. K., and Komura, T. (2011). A virtual reality dance training system using motion capture technology. IEEE Transactions on Learning Technologies, 4(2):187-195.

Clua, E. W. G. (2014). Jogos sérios aplicados a saúde. Journal of Health Informatics, 6.

Duarte, N., Postolache, O., and Scharcanski, J. (2014). Ksgphysio-kinect serious game for physiotherapy. In Electrical and Power Engineering (EPE), 2014 International Conference and Exposition on, pages 606-611. IEEE.

Estepa, A., Piriz, S. S., Albornoz, E., and Martínez, C. (2016). Development of a kinectbased exergaming system for motor rehabilitation in neurological disorders. In Journal of Physics: Conference Series, volume 705, page 012060. IOP Publishing. 
Fernandes, F. G., Santos, S. C., de Oliveira, L. C., Rodrigues, M. L., and Vita, S. S. B. V. (2014). Realidade virtual e aumentada aplicada em reabilitação fisioterapêutica utilizando o sensor kinect e dispositivos móveis. anais... volume 12, pages 1-6. Universidade Federal de Uberlândia.

Han, J., Shao, L., Xu, D., and Shotton, J. (2013). Enhanced computer vision with microsoft kinect sensor: A review. Cybernetics, IEEE Transactions on, 43(5):1318-1334.

Instruments, N. (2017). O que é o labview? Online. Disponível em: http://www.ni.com/newsletter/51141/pt. Acesso em: 12 mar. 2017.

Junior, V. D., Monteiro, C. B., Nakamura, R., Yojo, L. S., Araújo, L. V., and Nunes, F. L. (2013). Mover: Serious game aplicado a reabilitação motora usando sensor de movimento kinect.

Liu, T. T., Hsieh, C. T., Chung, R. C., and Wang, Y. S. (2013). Physical rehabilitation assistant system based on kinect. In Applied Mechanics and Materials, volume 284, pages 1686-1690. Trans Tech Publ.

Microsoft (2017). Kinect for windows sensor components and specifications. Online. Disponível em: https://msdn.microsoft.com/en-us/enus/library/jj131033.aspx. Acesso em: 28 jan. 2017.

Mira (2016). Mira - product. Online. Disponível em: http://www.mirarehab.com/product. Acesso em: 12 set. 2016.

Neuroathome (2016). Virtual reality rehabilitation. Online. Disponível em: http://www.neuroathome.com/en/. Acesso em: 08 dez. 2016.

Rehabilitation, V. R. (2017). Kinect rehabilitation with biofeedback. Online. Disponível em: http://www.virtual-reality-rehabilitation.com/. Acesso em: 22 jan. 2017.

Romano, R. G., Raia, F., Dias, I. R., and Assis, S. M. B. (2013). Tecnologia de games e reabilitação virtual: adaptação do software labview para captura dos dados do wii balance board. Millenium, (45):181-191.

Saini, S., Rambli, D. R. A., Sulaiman, S., Zakaria, M. N., and Shukri, S. R. M. (2012). A low-cost game framework for a home-based stroke rehabilitation system. In 2012 International Conference on Computer \& Information Science (ICCIS), volume 1, pages 55-60. IEEE.

Sousa, F. H. (2011). Uma revisão bibliográfica sobre a utilização do nintendo $囚$ wii como instrumento terapêutico e seus fatores de risco. Revista Espaço Acadêmico, 11(123):155-160.

Sousa, K. A., Neto, J. F. N., Silva, É. S., and Rodrigues, M. A. F. (2016). A gesture control system to support rehabilitation exercises. In Virtual and Augmented Reality (SVR), 2016 XVIII Symposium on, pages 170-177. IEEE.

Vendramini, M. D., Fernandes, A. M., and Paschoal, A. R. (2015). Serious games na saude: aplicação de dispositivos non-touch em atividades de fisioterapia. In: $X V$ Workshop de Informática Médica, Recife-PE.

Webb, J. and Ashley, J. (2012). Beginning Kinect Programming with the Microsoft Kinect SDK. Apress. 